\title{
Dental treatment of medically compromised children
}

\author{
Analysis of paediatric dental care provided under general anaesthesia and levels of dental disease in two hospitals
}

\section{A. Camilleri, G. Roberts, P. Ashley and B. Scheer Br Dent J 2004; 196: 219-223}

\section{Objective}

To compare the level of dental disease and the pattern of dental treatment under controlled airway general anaesthesia for ASA I and II children and ASA III and IV children in two hospitals.

\section{Design}

Prospective analysis.

\section{Setting}

Hospital and postgraduate dental teaching institution, UK 1996 2000.

\section{Subjects and methods}

During a four-year period (1996-2000), data were collected on children aged 1 to 16 years who were admitted for treatment of dental caries under general anaesthesia.

\section{Outcome measures}

Levels of dental disease (dmft/DMFT), treatment provided.

\section{Results}

A total of 515 ASA I and II and 430 ASA III and IV children were treated. The dmft and DMFT values of the ASA I and II children were significantly higher than those of the ASA III and IV children $(p=0.03)$. A significantly greater number of restorations and fissure sealants were carried out for permanent teeth in ASA III and IV children $(p<0.001)$. The number of extractions for both primary and permanent teeth was significantly greater in the ASA I and II group $(\mathrm{p}<0.05)$

\section{Conclusions}

The ASA III and IV children had significantly lower levels of dental caries than the ASA I and II children and received a higher level of preventive and restorative care.

\section{IN BRIEF}

- Previous published data have suggested that medically compromised children receiving dental treatment under GA have had more extractions and less restorations than the ASA I and II children undergoing similar procedures. This study demonstrates that this is not the case.

\section{COMMENT}

While the majority of children requiring dental treatment may be managed using local anaesthesia (either alone or in combination with conscious sedation), for some, the need for general anaesthesia remains. It has previously been suggested that medically compromised children receiving comprehensive dental care under general anaesthesia have more extractions and fewer restorations than ASA I and II children treated in this way. However, this study shows that ASA III and IV children undergoing treatment under general anaesthesia in a tertiary referral centre received a significantly higher level of preventive and restorative care and a significantly lower mean number of extractions than ASA I and II children treated in a neighbouring postgraduate dental teaching hospital. Interestingly, the ASA III and IV children were shown to have significantly lower levels of dental caries in both dentitions than ASA I and II children. Unsurprisingly (since treatment planning for comprehensive care under general anaesthesia has to be radical if recourse to further general anaesthetics is to be avoided), there was an overall predominance of extractions over restorations in both groups of children.

It would appear unlikely that the differences between groups are attributable to differences in treatment philosophy. The fact that there was a predominance of extractions over restorations in both groups of children would suggest that the overall approach to treatment was similar in both centres. Rather, it is more likely that the treatment received by the ASA III and IV children, as well as their relatively better dental status at the time of operation, is a reflection of constant surveillance and early referral to Consultantled services in Paediatric Dentistry. It is unfortunate that not all medically compromised children benefit from the heightened awareness of the importance of dental health and the Consultantled Paediatric Dentistry service offered by a major tertiary referral centre for paediatric specialties.

Dr Lindsay Hunter, Clinical Senior Lecturer in Paediatric Dentistry, University of Wales College of Medicine doi:10.1038/sj.bdj.4810986 